\title{
UN DIÁLOGO ENTRE DISCURSOS Y HECHOS: EL ACERCAMIENTO DEL MERCADO COMÚN DEL SUR (Mercosur) YLA ALIANZA DEL PACÍFICO (AP)
}

\author{
A DIALOGUE BETWEEN DISCOURSES AND FACTS: THE \\ RAPROCHEMENT BETWEEN THE MERCADO COMÚN \\ DEL SUR (MERCOSUR) AND THE PACIFIC ALLIANCE (PA)
}

UN DIALOGUE ENTRE LES DISCOURS ET LES FAITS: LE RAPPROCHEMENT ENTRE LE MARCHÉ COMMUN DU SUD (MERCOSUR) ET L'ALLIANCE DU PACIFIQUE (AP)

\author{
Mariana Aparicio Ramírez \\ UNAM FCPYS \\ aparicio.mariana@politicas.unam.mx \\ Gustavo Adolfo Islas Cadena \\ UNAM FCPYS \\ adogus11@gmail.com \\ IVÁN LóPEz MARTínEZ \\ UNAM FCPYS \\ ivanlopez@politicas.unam.mx
}

Resumen: El acercamiento entre la Alianza del Pacífico (AP) y el Mercosur ha sido un tema en discusión ante los virajes políticos que experimenta la región de América Latina. A pesar de los discursos oficiales, es posible identificar que no existe un interés o beneficio económico en la convergencia de ambos bloques, incluso con la existencia de diálogos formales como el Plan de Puerto Vallarta. Los hechos señalan que la propuesta responde más a intereses de actores políticos particulares que buscan cumplir sus objetivos de gobierno.

Palabras clave: Alianza del Pacífico (AP); Mercosur; diálogo económico; discurso político; integración económica.

Aвstract: The rapprochement between the Pacific Alliance (PA) and Mercosur has been a subject of discussion in light of the political changes experienced in the Latin America region. Regardless of official discourse, it is possible to 
identify that there is no interest or economic benefit in the convergence of the two blocks, even allowing for the existence of formal dialogues like the Puerto Vallarta Plan. The facts suggest that the proposal is more indicative of the interests of individual political actors seeking to meet their goals in government.

Keywords: Pacific Alliance (PA); Mercosur; economic dialogue; political discourse; economic integration.

\section{Traducción de Fionn Petch, CM Idiomas}

RÉSumÉ: Dans un contexte de virages politiques que traverse l'Amérique latine, le rapprochement entre l'Alliance du Pacifique (AP) et le Mercosur est un thème qui fait débat. Malgré les discours officiels, on peut voir que la convergence de ces deux blocs n'apporte aucun intérêt ou avantage économique, et ce même si des échanges formels tels que le Plan de Puerto Vallarta ont lieu. Les faits nous signalent qu'une telle intention tient davantage aux objectifs gouvernementaux que certains acteurs politiques spécifiques tentent de faire avancer par ce biais.

Mots clés: Alliance du Pacifique(AP); Mercosur; dialogue économique; discours politique; intégration économique.

Traducción de Ariel Elbaz, CM IDIOMAS

Fecha de recepción: febrero de 2020

Fecha de aceptación: octubre de 2020 


\section{INTRODUCCIÓN}

\section{$\mathrm{E}$}

$\mathrm{n}$ años recientes, el mundo ha pasado por una creciente alza de movimientos de corte conservador, ejemplo de ello es la llegada de Donald Trump a la presidencia de Estados Unidos en 2017; su lema de campaña, "Make America great again", pronosticaba la vuelta a modelos superados por su antecesor Barack Obama. Al otro lado del Atlántico, el Brexit triunfaba en las urnas británicas el 23 de junio de 2016, suceso que llevó a la renuncia de David Cameron y el ascenso de personajes como Teresa May y Boris Johnson al cargo de primer ministro, dedicando así sus labor a negociar la salida del Reino Unido de la Unión Europea, que se concretó el 31 de enero de 2020.

América Latina no fue ajena a esta tendencia, los casos más representativos fueron Argentina y Brasil. El primero contó con el mandato del empresario Mauricio Macri al frente de la Casa Rosada, de diciembre de 2015 hasta la vuelta al poder del kirchnerismo con Alberto Fernández, en diciembre de 2019; ${ }^{1}$ y el segundo, con el ascenso a la presidencia brasileña del evangelista Jair Bolsonaro a inicios de 2019. Ante esta coyuntura, la propuesta de acercamiento entre el Mercosur y la Alianza del Pacífico (AP) adquirió mayor relevancia para el desarrollo y alcance de los objetivos formulados en las políticas de sus respectivos gobiernos.

El propósito central de este escrito es describir cómo el viraje político de Brasil y Argentina ha sido crucial para el avance del diálogo entre la AP y el Mercosur. Para ello, en primer lugar, se describen los antecedentes clave de esta propuesta. Por un lado, la puesta en marcha con los gobiernos de Chile, Uruguay y Paraguay y, por otro, los virajes políticos experimentados en Brasil y Argentina. En segundo lugar, se analizan brevemente los postulados teóricos que permiten entender

${ }^{1}$ Para propósitos de análisis, esta investigación se centra solamente en el mandato de Mauricio Macri. 
cómo el factor político puede ser un determinante para aceptar o rechazar las propuestas de integración regional; además, se explora si hay complementariedad en la tendencia política de los gobiernos al frente de Brasil y Argentina, pues ambos cuentan con un elevado grado de influencia en el Mercosur. Posteriormente, se describe el intercambio comercial entre ambos bloques, los cuales sugieren un intercambio comercial mínimo para la propuesta de convergencia; previo al cierre, se explicará de manera general el panorama que enfrenta el futuro de este diálogo y, al final, se esbozan brevemente las reflexiones finales.

Antecedentes del diálogo. Uruguay, Paraguay y Chile

La AP significó el inicio de un nuevo modelo de integración en América Latina, ${ }^{2}$ en sus propias bases se define como un espacio de integración política, económica y comercial con el objetivo de convertir al bloque de países que lo forman (México, Colombia, Perú y Chile) en un espacio competitivo y atractivo para el acercamiento con la región de Asia-Pacífico, misma que describen como una de las principales en el marco económico actual. ${ }^{3}$

Orientada al libre mercado, el interés de múltiples países en ella es para lograr un acercamiento estratégico, en prime-

${ }^{2}$ José Briceño considera que la AP representa el regionalismo abierto en América Latina. Se le considera un modelo de integración que vela por una mayor liberalización comercial y una mejor promoción de la competitividad internacional. La AP se caracteriza por ser una alternativa al modelo de integración de proyectos como Mercosur, que sigue la línea del regionalismo posliberal promoviendo un aspecto más social que comercial. José Briceño Ruiz, "Estado, mercado y el modelo económico de la integración regional en América Latina, los casos de Mercosur y Alianza del Pacífico", en José Briceño Ruiz y Edgar Viera Posada (eds.), Repensar la integración en América Latina, Colombia, Universidad Cooperativa de Colombia, 2019, pp. 21-68.

${ }^{3}$ Alianza del Pacífico, ¿Qué es la Alianza Pacífico? (sitio de internet), https://alianzapacifico.net/que-es-la-alianza 
ra instancia a América Latina y, en segunda, a la región de Asia-Pacífico. Cuenta con un total de 59 Estados observadores que, si bien no tienen margen de maniobra en el organismo, sí pueden participar en los foros. Además, ese estatus les permite acercarse al proceso de adhesión a la AP una vez que cumplan los demás requisitos. ${ }^{4}$

Por su parte, el Mercado Común del Sur (Mercosur) es un bloque formado por Argentina, Brasil, Paraguay, Uruguay, Venezuela ${ }^{5}$ y Bolivia, ${ }^{6}$ y fue un proyecto de integración basado en el "respeto a los principios de Democracia y de Desarrollo Económico, impulsando una integración con rostro humano". 7

El 24 de noviembre de 2014, Michelle Bachelet, entonces presidenta de Chile, dio el discurso de apertura en el seminario "Diálogo sobre integración regional: Alianza del Pacífico y Mercosur", donde señaló la importancia de la integración en América Latina sin importar las bases ideológicas de cada proyecto:

Nuestra América Latina ha vivido un proceso común, pero caracterizado por la distancia más que la integración. Y si bien en los últimos años hemos avanzado bastante, es momento de que aunemos nuestros esfuerzos y emprendamos un camino de mayor cooperación en pro de una vida mejor para nuestras sociedades.

${ }^{4}$ Para consultar los requisitos de adhesión a la Alianza del Pacífico, véase: Acuerdo Marco de la Alianza del Pacífico, 17 de febrero de 2017, https:/ / alianzapacifico.net/download/acuerdo-marco-de-la-alianza-delpacifico

${ }^{5}$ La República Bolivariana de Venezuela se encuentra suspendida por la aplicación del Protocolo de Ushuaia (1998) cuestionando el Estado de democracia que vive ese país.

${ }^{6}$ El Estado plurinacional de Bolivia se encuentra en proceso de adhesión.

${ }^{7}$ Mercosur, ¿Qué es el Mercosur? (sitio de internet), https://www.mer cosur.int/quienes-somos/en-pocas-palabras 
[...] Y me parece tremendamente auspicioso saber que durante este diálogo, nuestros objetivos comunes se irán delimitando, articulando una perspectiva compartida entre Mercosur, un proceso histórico en la región, y la Alianza del Pacifico. ${ }^{8}$

En este contexto, Paraguay y Uruguay buscaron concretar un diálogo de acercamiento con la AP. Uruguay destaca por tener acuerdos de libre comercio con cada uno de los Estados parte ${ }^{9}$ de igual forma, su estatus en la AP desde 2012 es de Estado observador y, si bien cumple con los requisitos para solicitar la adhesión, sus declaraciones se limitan a reafirmar la cooperación con el bloque. ${ }^{10}$ De hecho, en cuanto al proceso de integración, la postura de Uruguay se decanta por la unión entre los bloques, pues comparte la visión de Michelle Bachelet respecto a desideologizar los procesos de integración en la región. ${ }^{11}$

${ }^{8}$ Michelle Bachelet, "Discurso de S.E. la presidenta de la República, Michelle Bachelet, al inaugurar Seminario 'Diálogo sobre integración regional: Alianza del Pacífico y Mercosur'”, Dirección de Prensa del Gobierno de Chile, 24 de noviembre de 2014, http://archivospresidencia les.archivonacional.cl/uploads/r/archivo-presidencia-de-la-republica $/ 6$ /6/b/66b5e5383881b2187c106b989fa32d4feed0dac9e59689a27f7780d 1201b4b07/_home_aristoteles_documentos_DIS_0350.pdf

${ }^{9}$ El Tratado de Libre Comercio con México, firmado el 15 de noviembre de 2003; con Chile tiene una desgravación total del universo arancelario; con Colombia existe el Tratado Mercosur, Colombia, Ecuador y Venezuela (Acuerdo de Complementación Económica, ACE 59) y el acuerdo Uruguay-Colombia de febrero de 2005; por último, con Perú está el Tratado de Libre Comercio de agosto de 2011. Isabel Clemente Batalla, Camilo López Burian y Diego Telias, "Uruguay y la Alianza del Pacífico: ¿repensando el modelo de inserción internacional?", en Cuadernos sobre Relaciones Internacionales, Regionalismo y Desarrollo, vol. 10, núm. 19, Venezuela, 2015, pp. 23-46.

${ }^{10} \mathrm{Ibid} .$, p. 37.

${ }^{11}$ Isabel Clemente Batalla, "Comercio y protección externa de Uruguay en la Cuenca del Pacífico", en Mariana Aparicio Ramírez y Tomás Milton Muñoz Bravo, Retos y oportunidades de la Alianza del Pacífico en el actual contexto internacional convulso, México, unAM, 2019, pp. 155-171. 
Por su parte, Paraguay tuvo un acercamiento con el gobierno chileno tras ser suspendido del Mercosur a raíz del golpe de Estado al expresidente Fernando Lugo (acontecimientos que ocasionaron la aplicación del Protocolo de Ushuaia). En un inicio, dicho país mostró apoyo al discurso de Bachelet en el que promueve la alianza entre AP y Mercosur. En materia de cooperación, se ha mostrado interesado en continuar con las pláticas del proceso de integración, no obstante, señaló que su mayor apoyo se dará a través de las conversaciones en el marco del Banco Interamericano de Desarrollo (BID). ${ }^{12}$

\section{El viraje político de Brasil y Argentina: ¿El nUevo CAMINO DEL MERCOSUR?}

En la primera década del siglo xx, el panorama político en América del Sur se caracterizó por un viraje contrario al que hoy se experimenta en la región. Como lo señala Constanza Moreira, "se instalaban gobiernos de izquierda o centro izquierda que hacían augurar, para muchos, el fin de la década neoliberal". ${ }^{13}$ Ejemplo de ello es que en Brasil asumía el poder Luiz Inácio Lula da Silva con el Partido de los Trabajadores (PT) y en Argentina, Néstor Kirchner, bajo la bandera del Frente para la Victoria (FPV). No obstante, a finales de la presente década se observó una nueva tendencia con el ascenso de gobiernos de derecha en el caso de Brasil y Argentina: el evangelista Jair Bolsonaro y el empresario Mauricio Macri, respectivamente; el modelo progresista parecía verse interrumpido.

12 "Chile y Paraguay impulsan la relación Mercosur/Alianza del Pacífico", MercoPress, 21 de abril de 2018, https:/ / es.mercopress.com/2018/ 04/21/chile-y-paraguay-impulsan-la-relacion-mercosur-alianza-del-pacifico

13 Constanza Moreira, "El largo ciclo del progresismo latinoamericano y su freno: los cambios políticos en América Latina de la última década (2003-2015)", Revista Brasileira de Ciências Sociais, vol. 32, núm. 93, 2019, p. 1, http:/ / www.scielo.br/pdf/rbcsoc/v32n93/0102-6909-rbcsoc-329311 2017.pdf 
Ante este viraje, la política exterior de ambos países sufrió cambios importantes, lo cual se observa en "el abandono o la relativización del concepto de autonomía como parámetro de la política exterior, la concepción de un escenario internacional como un ámbito de oportunidades, más que de asimetrías, y el privilegio de las relaciones Norte-Sur", ${ }^{14}$ pues implicó el abandono de relaciones autónomas entre los Estados parte del Mercosur, enfocando y priorizando el desarrollo de actividades orientadas al libre mercado.

Como ejemplo de lo anterior, se observa la firma del acuerdo de libre comercio entre el Mercosur y la Unión Europea después de veinte años de negociaciones, el cual fue calificado como un hecho histórico tanto por el gobierno brasileño como por el argentino. ${ }^{15}$ En esta línea, Jair Bolsonaro señaló que "[el acuerdo comercial] sucedería cuando ese gran bloque de América del Sur no se guiase más por la orientación ideológica, y sí por su orientación de libre comercio". ${ }^{16}$ En sintonía con esta declaración, Mauricio Macri expresó que el acuerdo significa "una gran oportunidad para apostar fuerte por la consolidación y modernización de nuestro bloque"; además, destacó que existe "[...] una agenda impostergable de competitividad para llevar el Mercosur al siglo xxi y obtener el máximo de beneficios de todos los pasos que estamos dando". Por último, invitó a "seguir concretando avances en la convergencia con la Alianza del Pacífico, que nos permitan construir una región productiva con costas sobre dos océanos y ser uno de los polos de crecimiento más diná-

14 Alejandro Frenkel y Diego Azzi, "Cambio y ajuste: la política exterior de Argentina y Brasil en un mundo en transición (2015-2017)", Colombia Internacional, núm. 96, 2018, p. 189, https://revistas.uniandes.edu. co/doi/pdf/10.7440/colombiaint 96.2018 .07

15 "La Unión Europea y el Mercosur logran un acuerdo comercial tras veinte años de negociación”, EFE, 28 de junio de 2019, https://www.efe. $\mathrm{com} /$ efe/america/portada/la-union-europea-y-el-mercosur-logran-unacuerdo-comercial-tras-veinte-anos-de-negociacion/20000064-4011791

${ }^{16}$ Loc. cit. 
micos del mundo". ${ }^{17}$ Sobre esta misma línea discursiva respecto al acuerdo con la Unión Europea, el presidente de la Comisión Europea, Jean-Claude Juncker, declaró que " $[\mathrm{e}] \mathrm{n}$ medio de tensiones comerciales internacionales, hoy transmitimos, junto con nuestros socios del Mercosur, una señal clara de que defendemos un comercio basado en normas. A través de este pacto comercial, los países del Mercosur han decidido abrir sus mercados a la UE". ${ }^{18}$ La revisión de estas declaraciones sugiere que el viraje político experimentado en Brasil y en Argentina permitió concretar, de manera positiva, las negociaciones que llevaban más de dos décadas sin resolución, pues gran parte de los avances se dieron en este periodo de cambio.

\section{LA IMPORTANCIA DEL FACTOR POLÍTICO EN LA INTEGRACIÓN ECONÓMICA}

En la extensa literatura sobre el regionalismo en los países en desarrollo se han señalado algunas de las dificultades que enfrentan estos países para concretar los procesos de integración a nivel regional. Como primer punto, se observa que el entorno económico no necesariamente permite una correcta convergencia entre iguales, lo cual se sustenta en las fallas que han tenido la teoría clásica del comercio, la teoría del desarrollo competitivo y la de la dependencia, al proponer modelos a seguir para los países en desarrollo. ${ }^{19}$

17 Gobierno de Argentina, "Macri, sobre el acuerdo Mercosur-UE: 'Es fruto de ese trabajo colectivo', Argentina, Casa Rosada Presidencia, 2019, https:/ / www.casarosada.gob.ar/slider-principal/45831-macri-sobre-elacuerdo-mercosur-ue-es-fruto-de-ese-trabajo-colectivo

${ }^{18}$ Delegation of the European Union to Chile, "La uE y el Mercosur llegan a un acuerdo en materia de comercio", Bruselas, Comisión Europea, 28 de junio de 2019, https://eeas.europa.eu/delegations/chile/ 64865/la-ue-y-el-mercosur-llegan-un-acuerdo-en-materia-de-comercio_uz

19 András Inotai propone que las fallas de cada teoría han ocurrido debido a que los hechos en la realidad no empatan con los postulados 
Un segundo punto se refiere al ámbito político como otro factor que ha logrado frenar los procesos de integración en estos países. Sobre esta línea, András Inotai señala que la orientación en política económica de los países (ya sea a favor del libre mercado o de la administración centralizada), en conjunto con la falta de fortaleza de sus instituciones y los conflictos civiles, dificulta el trato y las negociaciones entre las partes. ${ }^{20}$ De igual manera, Langhammer y Hiemenz proponen que, en ciertas circunstancias, los países aceptan los procesos de integración con el fin de obtener beneficios políticos, tales como seguridad. ${ }^{21}$

En este debate, Schiff y Winters reiteran que la aceptación o rechazo de los procesos de integración pueden analizarse desde las perspectivas ya mencionadas: económica y política. ${ }^{22}$ La primera se basa principalmente en la búsqueda

teóricos: 1) para el caso de la teoría clásica del comercio, los datos econométricos de los países en desarrollo, asociados a algún proyecto de integración regional, no necesariamente señalan una mejora deseable para sus economías; 2) la teoría de la competitividad muestra que las condiciones de estas economías a escala son aprovechables para que los países en desarrollo mejoren sus industrias locales y lograr así una mejor inserción en el mercado internacional, sin embargo, la mayoría de las industrias de estas economías no muestran un nivel favorable de competitividad internacional; 3) la teoría de la dependencia señala que la integración en modelos regionales permite reducir la dependencia hacia los países desarrollados, no obstante, esta dependencia no puede reducirse en la mayoría de los casos debido a que las relaciones intrarregionales no logran cubrir todos los ámbitos. Sobre este aspecto, se recomienda consultar: András Inotai, "Regional integration among developing countries, revisited", Policy Research Working Paper Series, núm. 643, 1991, The World Bank, pp. 4-9, http://documents1.worldbank.org/curated/en/647131468739252693/ pdf/multi0page.pdf

${ }^{20}$ Ibid., p. 19.

${ }^{21}$ RolfJ. Langhammer y Ulrich Hiemenz, "Regional integration among developing countries: opportunities, obstacles and options", Kieler Studien, núm. 232 (1990), pp. 16-17, https://www.econstor.eu/handle/10 419/416

${ }^{22}$ Maurice Schiff y Alan L. Winters, Regional integration and development, Washington, D.C., The World Bank Group, 2003, pp. 1-29, http:// documents.worldbank.org/curated/en/263681468780873762/Regionalintegration-and-development 
de apertura de mercados entre las partes con el fin de alcanzar un crecimiento económico a través de la actividad comercial y la entrada de inversiones. Además, la consolidación de estos modelos permite crear centros atractivos para la inversión extranjera. Por otro lado, en la segunda, la integración con fines políticos señala que su desarrollo puede orientarse a reducir las tensiones entre los miembros, a la par que promover la paz y la democracia. ${ }^{23}$

Sobre la integración por razones políticas, Christina Schneider elabora un panorama sobre la política económica en los acuerdos regionales. La autora identifica que está presente la influencia de diferentes actores; por un lado, los grupos domésticos que logran incidir en la dinamización de los procesos de integración regional, valiéndose del entramado institucional que permite que sus propuestas lleguen a los gobernan$\operatorname{tes}^{24} y$, por otro, considera que la posición del mandatario puede influir en promover u obstaculizar la integración a los modelos regionales que se le presentan. ${ }^{25}$

En el caso de América Latina, los procesos de integración han transitado por momentos clave donde el elemento político ha sido un determinante mayor. Desde este enfoque, se han identificado una serie de iniciativas regionales estudiadas bajo las denominadas "olas" de integración: 1) el regionalismo desarrollista impulsado por la Comisión Económica para América Latina y el Caribe (Cepal); 2) el regionalismo abierto con ejemplos como el Mercosur y el Mercado Común Centroamericano (MCCA) $;{ }^{26}$ y 3) el regionalismo pos-

23 Para mayor detalle sobre cómo la integración regional se desarrolla en torno a un enfoque político, se recomienda consultar el capítulo 7, "Regional Integration politics", en Schiff y Winters, Ibid.

${ }^{24}$ Christina J. Schneider, "The Political Economy of Regional Integration”, Annual Review of Political Science, vol. 20, 2017, p. 234, https://www. annualreviews.org/doi/full/10.1146/annurev-polisci-051215-023006

${ }^{25}$ Loc. cit.

${ }^{26}$ En su inicio, el Mercosur es considerado un modelo de integración de regionalismo abierto, no obstante, a raíz de los gobiernos progresistas en la región, adoptó una visión de regionalismo posliberal, características que se mencionan en los apartados posteriores. 
liberal o poshegémonico con la ALBA (Alianza Bolivariana para los pueblos de Nuestra América) y la Unasur (Unión de Naciones Suramericanas). ${ }^{27}$ En esta clasificación, la AP se posiciona como una propuesta de integración que busca retomar el regionalismo abierto teniendo como bases la desregulación económica y la liberalización de mercados entre los miembros. ${ }^{28}$

En la línea del análisis del factor político como eje central de este artículo, debe considerarse el peso de los miembros de un proceso de integración, pues no todos comparten la misma capacidad de influencia en la toma de decisiones. En el Mercosur, se propone analizar Brasil y Argentina como los países con mayor peso en este proceso del organismo. Para el caso de Brasil, Carvahlo y Carolina de Souza lo consideran un region-builder ${ }^{29}$ que enfrenta los distintos desafíos que representan los procesos de integración en América Latina. ${ }^{30}$ Por su parte, Argentina históricamente ha tenido un papel importante como aliado de Brasil en la evolución de los procesos de integración en la región. Como ejemplo se tiene la formación de la Asociación Latinoamericana de Libre Comercio (Alalc) en 1960 impulsado en parte por el clima de cooperación entre Kubitschek y Frondozi (presidente de Brasil y Argentina respectivamente) ${ }^{31} \mathrm{y}$, por otro lado, la

${ }^{27}$ Eduardo Pastrana y Rafael Castro, "La Alianza del Pacífico, un eje articulador del interregionalismo pos-TPP”, Anuario de la integración regional de América Latina y el Caribe, núm. 14, 2017, pp. 72-73, http://www. cries.org/wp-content/uploads/2018/04/Anuario-2017-final.pdf

${ }^{28}$ Loc. cit.

${ }^{29}$ Un region-builder se considera "aquel país que dará el primer paso en la construcción de una determinada región”. Este concepto se retoma de la literatura del region-building de Luk Langenhove, Building Regions: The Regionalization of the World Order, Farnham, Ashgate, 2011. Para mayor información en torno al concepto, véase: Vinícius de Carvalho Castro Madureira y Carolina P. de Souza, "Brasil y la integración regional", Estado E Comunes, Revista de políticas y problemas públicos, vol. 2, núm. 9, 2019, p. 201, https://doi.org/10.37228/estado_comunes.v2.n9.2019.125

${ }^{30}$ Ibid., p. 209.

${ }^{31}$ Javier A. Vadell y Clarisa Giaccaglia, "El rol de Brasil en el regionalismo latinoamericano: la apuesta por una inserción internacional solita- 
creación del Mercosur en un contexto posdictadura, donde se fomenta la visión democrática en lo político y la liberal en lo económico, aceptando así las bases del regionalismo abierto. ${ }^{32}$ Como se mencionó en la sección previa, el entendimiento entre ambos países vuelve a mostrarse con la transición que experimenta el Mercosur durante los gobiernos progresistas. ${ }^{33}$ En este sentido, la propuesta de convergencia entre dos bloques como AP y Mercosur despierta interés para un análisis que permita entender el incentivo que detonó la búsqueda de esta asociación, pues los hechos sugieren que el factor político tiene mayor peso que el económico.

Cabe resaltar que, entre ambos organismos, Mercosur cuenta con una historia más larga en el ámbito político debido a su creación en 1991, mientras que la AP, dado su poco tiempo en el escenario, aún no presenta características que permitan efectuar un análisis más profundo. Además, las cuestiones políticas en los países del Mercosur influyen en las relaciones con otros organismos. Por ejemplo, el aislacionismo y distanciamiento integracionista durante el periodo

ria y unilateral”, Foro Internacional, vol. 60, núm. 3 (2020), p. 1044, https:/ / doi.org/10.24201/fi.v60i3.2770

32 De acuerdo con María M. Llairó, el regionalismo abierto se caracteriza por priorizar una integración de carácter económico a través de estrategias implementadas por el comercio mundial, en coincidencia con los gobiernos en turno que se adherían a los principios del libre mercado, con el fin de aumentar la competitividad de los países latinoamericanos en un mundo globalizado. Para mayor información consultar: María de Monserrat Llairó, "Los nuevos desafíos y ejes de poder de la integración latinoamericana: la dualidad Mercosur-Alianza del Pacífico (2010-2017)", Anuario Latinoamericano-Ciencias Políticas y Relaciones Internacionales, vol. 7 (2019), p. 113, https://doi.org/10.17951/al.2019.7.111-129

33 Otra forma de apreciar esta coordinación ante los cambios políticos en el Mercosur es a partir de los ingresos y suspensiones en el organismo. Durante el periodo progresista se tuvo la suspensión de Paraguay tras el juicio político de Fernando Lugo; más tarde, en 2018, la suspensión de Venezuela con la vuelta de la derecha en los gobiernos de Argentina y Brasil. Alberto van Klaveren, "El eterno retorno del regionalismo latinoamericano", Revista Nueva Sociedad, núm. 275 (2018), p. 11, https://nuso. org/media/articles/downloads/3.TC_van_Klaveren_275.pdf 
progresista de los miembros del Mercosur ilustra el rechazo a la AP, por tener en sus inicios un predominio de gobiernos abiertos a la inserción económica internacional. ${ }^{34}$ Más tarde, con el viraje político en Argentina y Brasil, sus críticas a la AP por su relación con Estados Unidos disminuyeron en pro de potenciar el acercamiento entre ambos grupos. ${ }^{35}$

En suma, se muestra la importancia del factor político como motor para los procesos de integración como un determinante que en este proyecto de convergencia, incluso puede ser un mayor incentivo que el factor económico.

\section{EVANGELISTAS Y EMPRESARIOS DE MERCADO: LA NUEVA DINÁMICA EN MERCOSUR}

En la sección anterior se estableció la importancia del factor político como clave para concretar un proceso de integración. En este sentido, es posible sugerir que el interés político hacia estas propuestas integracionistas está vinculado con un proyecto de gobierno más que de Estado, de acuerdo con intereses y objetivos respecto a la inserción económica internacional. Todo ello supone que un viraje del gobierno en Argentina y Brasil representa un cambio en su política de gobierno en lo que atañe a los procesos de integración. Por lo tanto, es importante establecer cómo la tendencia hacia la izquierda o la derecha ${ }^{36}$ de los gobiernos explica los cambios

${ }^{34}$ Rebeca Rodríguez Minor, "Alianza del Pacífico vs. Mercosur. ¿Integración o exclusión regional?”, Revista de Relaciones Internacionales, núm. 125 (2016), p. 135, http://www.revistas.unam.mx/index.php/rri/article/view $/ 58600$

${ }^{35}$ Alberto Martínez-Castillo, "The Pacific Alliance and Mercosur: Two Approaches to Economic Regional Integration", Papel Politico, vol. 21, núm. 2, 2016, p. 487, https://doi.org/10.11144/Javeriana.papo21-2.apmc

${ }^{36}$ La dualidad de izquierda y derecha, de acuerdo con Bobbio, se puede entender a partir de la "actitud que asumen los hombres que viven en sociedad frente al ideal de la igualdad". En la actualidad, es posible identificar a la derecha con gobiernos más cercanos a la ideología neoliberal, mientras que la izquierda se ubica en gobiernos que priorizan el 
en la postura de aceptación o rechazo en la convergencia de la AP y Mercosur. Para este propósito, se retoma el modelo de dos ejes -el cruce entre espectro político de derecha e izquierda y posturas respecto a la globalización a favor o en contra- de José Antonio Sanahuja, en el que propone cuatro posibles posiciones vis à vis los acontecimientos de política internacional: 1) globalistas de derecha; 2) globalistas progresistas; 3) desglobalizadores (antiglobalización e izquierda) y nuevos patriotas (antiglobalización y derecha). ${ }^{37}$

En el caso de Argentina, Sanahuja clasifica al gobierno de Macri como globalista de derecha, al ser "favorable a la democracia liberal, el libre comercio y la empresa privada: proclives a profundizar la integración económica global, y partidarios del statu quo de la globalización". ${ }^{38}$ Hay que recordar que el presidente argentino, desde el comienzo de su mandato, mostró una postura liberal en el ámbito económico y conservadora en lo político. ${ }^{39}$

Por su parte, desde su campaña electoral Jair Bolsonaro mostró una fuerte postura antiglobalista, similar a la del presidente de Estados Unidos, Donald Trump. Por tal motivo, ha sido categorizado por Sanahuja en el grupo "nuevos patriotas", los cuales "socialmente, agrupan a perdedores -reales o

aspecto social de su población. Norberto Bobbio, Derecha e izquierda: razones y significados de una distinción política, Madrid, Taurus, 1996.

${ }^{37}$ Los globalistas de derecha están a favor de la democracia liberal, el libre comercio y la iniciativa privada; los globalistas progresistas favorecen la regularización de la globalización a través de normas regionales que garanticen derechos básicos; los desglobalizadores son movimientos de izquierda opuestos a la globalización; finalmente, los nuevos patriotas se oponen al libre comercio y critican los organismos multilaterales al considerarse los verdaderos perdedores de la globalización, socialmente son nacionalistas con tintes xenófobos y racistas. Véase José Antonio Sanahuja, "El ascenso global de la ultraderecha y el nacionalismo: crisis de la globalización, el regionalismo y el orden liberal”, en Josette Altmann (ed.), América Latina frente a la reconfiguración global, Costa Rica, Flacso, 2019, pp. 31-64.

${ }^{38}$ Ibid., pp. 54-56

${ }^{39}$ Alejandro Frenkel y Diego Azzi, art. cit., p. 179. 
autopercibidos- de la globalización, como clases medias y medias bajas afectadas por el desempleo y la desindustrialización; y a sectores rurales y de mayor edad que rechazan el cosmopolitismo y el multiculturalismo". ${ }^{40}$ Los "nuevos patriotas", en cuestiones de política comercial, pueden tener diferentes enfoques, por ejemplo, el Frente Nacional en Francia se considera proteccionista; y los brexiteers muestran la continuidad del modelo económico liberal en solitario. ${ }^{41}$ En el caso de Brasil, el presidente Bolsonaro busca implementar una política económica neoliberal. Para lograr su cometido ha confiado la economía a Paulo Guedes, garante de las reformas económicas ultraliberales que prometió. ${ }^{42}$

Aunque la política exterior de Bolsonaro sugiera en primera instancia una visión unilateral y separada de la cuestión regional, el gobierno no es ajeno al Mercosur, pues representa para él una vía de acceso a la tan deseada inserción en el mercado global.

No obstante, recurrir al Mercosur como instrumento que permita el acceso a nuevos mercados requiere una compatibilidad entre las posturas económicas de los países miembros del esquema de integración. La declaración emitida por Paulo Guedes para el diario argentino El Cronista ilustra la necesidad de un entendimiento entre las partes:

[E]1 Mercosur parece ir ahora en el sentido que acabo de describirle (teniendo como ejes el crecimiento económico, mayor apertura, competitividad y productividad), pero no tengo certeza que siga así porque tenemos elecciones ahora en la Argentina. Ojalá el Mercosur siga siendo un instrumento para la modernización y la apertura de las economías. ${ }^{43}$

40 José Antonio Sanahuja, art. cit., pp. 55-56.

41 Ibid., p. 56.

42 Gilberto Rodrigues, “¿El Trump del trópico? Política exterior de ultraderecha en Brasil”, Análisis Carolina, núm. 6, (2019), p. 6, https:// doi.org/10.33960/AC_06pt.2019

43 Ezequiel Chabay, "Guedes, en exclusiva: qué pasa con el "peso real', por qué votaría a Macri y qué pasará si vuelve CFK”, El Cronista, 16 de 
Las palabras del ministro brasileño reflejaron las buenas relaciones político-económicas, entre los gobiernos de Macri y Bolsonaro, pues la postura que ambos gobiernos comparten respecto a la apertura económica permitió, al menos en ese tiempo, un viraje en el Mercosur.

Esta misma idea la comparte el gobierno argentino. Durante su campaña, Mauricio Macri promulgó la búsqueda de un cambio, rechazando los proyectos populistas y manifestando el regreso al mundo como única alternativa. ${ }^{44} \mathrm{~A}$ su llegada a la Casa Rosada, Mauricio Macri mostró una nueva visión económica-comercial; ${ }^{45}$ en un inicio adelantó la intención de flexibilizar el Mercosur y acercarse a la AP. Las visitas a Colombia y Perú, el acercamiento de Argentina a Chile como socio estratégico, ${ }^{46}$ la llegada a Buenos Aires del presidente Peña Nieto y la anexión de Argentina como país observador de la AP confirman esta tendencia. ${ }^{47}$

julio de 2019, https://www.cronista.com/economiapolitica/Guedes-enexclusiva-que-pasa-con-el-peso-real-por-que-votaria-a-Macri-y-que-pasarasi-vuelve-CFK-20190716-0084.html

${ }^{44}$ Laura María D’Alesio, "La 'nueva derecha' y su política exterior. Los primeros tres años de gestión del gobierno de Mauricio Macri”, Perspectivas Revista de Ciencias Sociales, Año 4, núm. 8 (2019), pp. 204-205, https://doi.org/10.35305/prcs.v0i8.58

${ }^{45}$ En esta misma línea se puede consultar el capítulo del entonces embajador de Argentina acreditado en México. Véase Daniel Chuburu, "La convergencia Mercosur, Alianza del Pacífico desde la perspectiva argentina: los desafíos de profundizar la integración regional en la actualidad”, en Mariana Aparicio Ramírez y Tomás Milton Muñoz Bravo, Retos y oportunidades de la Alianza del Pacifico en el actual contexto internacional convulso, México, unam, 2019, pp. 41-48.

${ }^{46} \mathrm{Al}$ respecto del acercamiento con Chile, se recomienda consultar a Francisco Castaño, "La inserción internacional argentina durante el gobierno de Macri”, ponencia presentada en el VIII Encuentro del CERPI y la VI Jornada del Censud, unlP, Argentina, 2017, p. 3, http://sedici.unlp.edu. ar/handle/10915/64890; y a René Hernández, "La encrucijada de la integración regional y el nuevo regionalismo", Revista Estudios de Políticas Públicas, vol. 7 (2018), p. 154, https://doi.org/10.5354/0719-6296.2018.50427

47 Anabella, Busso y Julieta Zelicovich, "El gobierno de Mauricio Macri y la integración regional: ¿desde el Mercosur a la Alianza del Pacífi- 
Con lo anterior se establece que las acciones del jefe de Estado argentino, en convergencia con las del gobierno brasileño, proponían la liberación comercial del bloque regional. En esta línea, se observa que existe la afinidad ideológica necesaria entre ambas administraciones sudamericanas.

La idea de los gobiernos de Bolsonaro y Macri sobre el futuro del Mercosur se estableció formalmente en la declaración conjunta emitida el 16 de enero de 2019 en Brasilia, durante la visita del presidente argentino a Brasil, en la cual se señala que:

[L] os presidentes [Bolsonaro y Macri] decidieron trabajar durante sus consecutivas presidencias pro tempore, en 2019, para rever la tarifa externa común, mejorar el acceso a mercados y avanzar en facilitación de comercio y convergencia regulatoria. En el plano externo, acordaron impulsar las negociaciones más promisoras ya en curso y evaluar el inicio de nuevas negociaciones con otros socios. ${ }^{48}$

Posteriormente, en la visita del mandatario brasileño a Buenos Aires en junio del mismo año, ambos gobernantes mantuvieron esta línea mediante una nueva declaración conjunta en la que:

Ratificaron la voluntad política de Argentina y Brasil en fortalecer y modernizar el Mercosur priorizando su reforma institucional y el intercambio comercial intra Mercosur, así como la inserción internacional del bloque a través de la moderniza-

co?”, Conjunta Austral, vol. 7, núm. 37, 2016, p. 19, https://doi.org/10.22 456/2178-8839.66725

48 Ministerio de Relaciones Exteriores de Brasil, "Declaración conjunta emitida en el marco de la visita de trabajo a Brasil del Presidente de la Nación Argentina, Mauricio Macri-Brasilia”, 16 de enero de 2019, Ministerio de Relaciones Exteriores de Brasil, 16 de ene de 2019, http:// www.itamaraty.gov.br/es/notas-a-la-prensa/19964-declaracion-conjuntaemitida-en-el-marco-de-la-visita-de-trabajo-a-brasil-del-presidente-de-lanacion-argentina-mauricio-macri-brasilia-16-de-enero-de-2019 
ción de su estructura arancelaria y de la conclusión de la negociación de los acuerdos comerciales en curso. ${ }^{49}$

De igual manera, durante esta reunión de jefes de Estado, se indicó a los equipos negociadores que, en conjunto con los de Paraguay y Uruguay se intensificarían las negociaciones comerciales con la Unión Europea, la Asociación Europea de Libre Comercio (EFTA por sus siglas en inglés) y Canadá, además de seguir con las negociaciones comerciales iniciadas en 2018 con Corea del Sur y Singapur. ${ }^{50}$

Las acciones anteriores muestran que el Mercosur figura como un instrumento potencial que permite a los gobiernos de Macri y Bolsonaro concretar su política de gobierno para alcanzar nuevos socios externos y potenciar los mercados regionales. De esta manera, se logra entender cómo el cambio político en ambos países fungió como un factor clave que permite aceptar las propuestas de integración para así cumplir con sus políticas de su gobierno.

INFORME DEL BID: EL ASPECTO ECONÓMICO DEL DIÁLOGO Mercosur-AP

Si bien el objetivo central de este trabajo no es analizar los aspectos económicos de esta convergencia, resulta relevante exponer los indicadores comerciales entre las partes, con miras a ilustrar la predominancia del factor político y descartar el factor económico como determinante en esta propuesta.

La búsqueda de una convergencia entre la AP y Mercosur se vio plasmada en 2018 con el Informe del Diálogo de Alto

${ }^{49}$ Ministerio de Relaciones Exteriores de Brasil, "Declaración Conjunta Presidencial en ocasión de la visita de Estado del Presidente Jair Bolsonaro a Buenos Aires", Ministerio de Relaciones Exteriores de Brasil, 6 de junio de 2019, http://www.itamaraty.gov.br/es/notas-a-la-prensa/20483declaracion-conjunta-presidencial-en-ocasion-de-la-visita-de-estado-delpresidente-jair-bolsonaro-a-buenos-aires

${ }^{50}$ Loc. cit. 
Nivel del Banco Interamericano de Desarrollo. ${ }^{51}$ En él se establecen los temas a seguir (cadenas regionales de valor/acumulación de origen, facilitación del comercio/ventanillas únicas de comercio exterior, cooperación aduanera, promoción comercial y pymes, barreras no arancelarias y facilitación del comercio de servicios) para una posible integración entre los dos bloques comerciales, poniendo el énfasis en incrementar la facilitación del comercio y potenciar las cadenas globales de valor.

En este informe se reconoce la existencia de una baja interacción comercial entre los países de ambas organizaciones, dado que "la Alianza del Pacífico fue destino de 6.9\% de las exportaciones del Mercosur y origen de $5.7 \%$ de sus importaciones en 2016. Como contrapartida, el Mercosur fue destino de $2.3 \%$ de las exportaciones de la Alianza del Pacífico y origen de $3.9 \%$ de sus importaciones" ${ }^{52}$ En ambos casos se observa que hay mercados poco atractivos para los dos bloques.

Para comprobar la existencia del poco dinamismo comercial entre estos bloques regionales, se analiza el intercambio comercial (exportaciones e importaciones) de cuatro países miembros del Mercosur ${ }^{53}$ y los cuatro países originales de la AP. Para ello, se tomó como referencia la base de datos Perfiles comerciales 2019, de la Organización Mundial del Comercio (OMC). ${ }^{54}$

${ }^{51}$ Instituto para la Integración de América Latina y el Caribe, Mercosur-Alianza del Pacífico: informe del Diálogo de Alto Nivel: una agenda positiva para la integración, BID, marzo de 2019, https:/ / publications.iadb.org/es/ publicacion/14106/mercosur-alianza-del-pacifico-informe-del-dialogode-alto-nivel-una-agenda

${ }^{52}$ Loc. cit.

53 Venezuela fue descartado de la selección debido a su suspensión en el Mercosur. Igualmente se descartó Bolivia porque aún no concreta en su totalidad el proceso de adhesión al bloque.

54 Véase Organización Mundial del Comercio, Perfiles comerciales 2019, oмc, 2019, https://www.wto.org/spanish/res_s/booksp_s/trade_profiles 19_s.pdf 
En los cuadros 1 y 2 se observa que Chile es el único miembro de la AP que funge como uno de los cinco principales destinos de las exportaciones de mercancías de Argentina y Paraguay, ocupando el quinto lugar como receptor, con $4.9 \%$ y $6.5 \%$ respectivamente.

\section{CuAdro 1}

Principales destinos de exportaciones e importaciones: Argentina

\begin{tabular}{lllll}
\hline Argentina & \multicolumn{2}{c}{ Exportaciones } & \multicolumn{2}{c}{ Importaciones } \\
\hline Mercancías & Brasil & $18 \%$ & Brasil & $23.8 \%$ \\
& Unión Europea & $15 \%$ & China & $18.4 \%$ \\
& Estados Unidos & $6.9 \%$ & Unión Europea & $17.2 \%$ \\
& China & $6.8 \%$ & Estados Unidos & $11.8 \%$ \\
& Chile & $\mathbf{4 . 9 \%}$ & Paraguay & $3.3 \%$ \\
\hline
\end{tabular}

Fuente: elaboración propia con base en los datos de Perfiles comerciales 2019, de la Organización Mundial del Comercio (омC).

\section{Cuadro 2}

Principales destinos de exportaciones e importaciones:

Paraguay

\begin{tabular}{clrlr}
\hline Paraguay & \multicolumn{2}{c}{ Exportaciones } & \multicolumn{2}{c}{ Importaciones } \\
\hline Mercancías & Brasil & $31.1 \%$ & China & $28.2 \%$ \\
& Argentina & $25.5 \%$ & Brasil & $22.3 \%$ \\
& Unión Europea & $8.7 \%$ & Unión Europea & $11.4 \%$ \\
& Rusia & $8.4 \%$ & Argentina & $10 \%$ \\
& Chile & $\mathbf{6 . 5 \%}$ & Estados Unidos & $7.8 \%$ \\
\hline
\end{tabular}

Fuente: elaboración propia con base en los datos de Perfiles comerciales 2019, de la Organización Mundial del Comercio (OMC).

Por su parte, el cuadro 3 indica los destinos de exportaciones e importaciones para el caso de Brasil, mientras que el cuadro 4 contiene los datos de Uruguay. Ambos, en conjunto 
con los datos antes expuestos, muestran que, salvo el caso chileno, ninguna de las economías de la Alianza Pacífico destaca en el rubro de exportaciones o importaciones, reafirmando con ello la idea del poco dinamismo comercial entre ambos bloques de integración.

\section{CuAdro 3}

Principales destinos de exportaciones e importaciones: Brasil

\begin{tabular}{clrlr}
\hline Brasil & \multicolumn{2}{c}{ Exportaciones } & \multicolumn{2}{c}{ Importaciones } \\
\hline Mercancías & China & $21.8 \%$ & Unión Europea & $21.2 \%$ \\
& Unión Europea & $16 \%$ & China & $18.1 \%$ \\
& Estados Unidos & $12.5 \%$ & Estados Unidos & $16.6 \%$ \\
& Argentina & $8.2 \%$ & Argentina & $6.2 \%$ \\
& Japón & $2.4 \%$ & República de Corea & $3.5 \%$ \\
\hline
\end{tabular}

Fuente: elaboración propia con base en los datos de Perfiles comerciales 2019, de la Organización Mundial del Comercio (омC).

\section{CuAdro 4}

Principales destinos de exportaciones e importaciones: Uruguay

\begin{tabular}{clrlr}
\hline Uruguay & \multicolumn{2}{c}{ Exportaciones } & \multicolumn{2}{c}{ Importaciones } \\
\hline Mercancías & China & $18.8 \%$ & China & $20 \%$ \\
& Brasil & $16.5 \%$ & Brasil & $19.5 \%$ \\
& Unión Europea & $11 \%$ & Unión Europea & $15.5 \%$ \\
& Estados Unidos & $5.8 \%$ & Argentina & $12.6 \%$ \\
& Argentina & $5.5 \%$ & Estados Unidos & $10.9 \%$ \\
\hline
\end{tabular}

Fuente: elaboración propia con base en los datos de Perfiles comerciales 2019, de la Organización Mundial del Comercio (oMc).

Desde la perspectiva de los países de AP se observa que, con base en los datos del cuadro 5, el único país del Mercosur que figura en alguno de los rubros comerciales es Brasil, que 
ocupa el quinto lugar en exportaciones para el caso de México donde representa únicamente $1 \%$.

\section{Cuadro 5}

Principales destinos de exportaciones e importaciones:

\section{México}

\begin{tabular}{clrlr}
\hline México & \multicolumn{2}{c}{ Exportaciones } & \multicolumn{2}{c}{ Importaciones } \\
\hline Mercancías & Estados Unidos & $76.5 \%$ & Estados Unidos & $46.6 \%$ \\
& Unión Europea & $4.7 \%$ & China & $18 \%$ \\
& Canadá & $3.1 \%$ & Unión Europea & $11.4 \%$ \\
& China & $1.6 \%$ & Japón & $3.9 \%$ \\
& Brasil & $\mathbf{1 \%}$ & República de Corea & $3.6 \%$ \\
\hline
\end{tabular}

Fuente: elaboración propia con base en los datos de Perfiles comerciales 2019, de la Organización Mundial del Comercio (омC).

En cuestión de importaciones (véanse cuadros 6, 7 y 8), Brasil representa el origen de $9 \%, 5.5 \%$ y $6.2 \%$ de los productos que ingresan a territorio de Chile, Colombia y Perú, respectivamente. Además, Argentina solamente aparece en el caso de Chile, donde representa el origen de $4.6 \%$ de las mercancías que recibe ese país.

\section{Cuadro 6}

Principales destinos de exportaciones e importaciones: Chile

\begin{tabular}{llrlr}
\hline \multicolumn{1}{c}{ Chile } & \multicolumn{2}{c}{ Exportaciones } & \multicolumn{2}{c}{ Importaciones } \\
\hline Mercancía & China & $33.5 \%$ & China & $23.6 \%$ \\
& Estados Unidos & $13.8 \%$ & Estados Unidos & $18.9 \%$ \\
& Unión Europea & $11.6 \%$ & Unión Europea & $14.9 \%$ \\
& Japón & $9.3 \%$ & Brasil & $\mathbf{9 \%}$ \\
& República de Corea & $5.8 \%$ & Argentina & $\mathbf{4 . 6 \%}$ \\
\hline
\end{tabular}

Fuente: elaboración propia con base en los datos de Perfiles comerciales 2019, de la Organización Mundial del Comercio (OMC). 


\section{CUADRo 7}

Principales destinos de exportaciones e importaciones:

Colombia

\begin{tabular}{clrlr}
\hline Colombia & \multicolumn{2}{c}{ Exportaciones } & \multicolumn{2}{c}{ Importaciones } \\
\hline Mercancías & Estados Unidos & $27.1 \%$ & Estados Unidos & $25.6 \%$ \\
& Unión Europea & $11.8 \%$ & China & $20.6 \%$ \\
& China & $9.7 \%$ & Unión Europea & $14.8 \%$ \\
& Panamá & $7.3 \%$ & México & $7.7 \%$ \\
& Ecuador & $4.4 \%$ & Brasil & $\mathbf{5 . 5 \%}$ \\
\hline
\end{tabular}

Fuente: elaboración propia con base en los datos de Perfiles comerciales 2019, de la Organización Mundial del Comercio (OMC).

\section{Cuadro 8}

Principales destinos de exportaciones e importaciones: Perú

\begin{tabular}{llrlr}
\hline Perú & Exportaciones & \multicolumn{3}{l}{ Importaciones } \\
\hline Mercancías & China & $26.3 \%$ & China & $22.3 \%$ \\
& Estados Unidos & $15.7 \%$ & Estados Unidos & $20.3 \%$ \\
& Unión Europea & $14.7 \%$ & Unión Europea & $12.2 \%$ \\
& Suiza & $5.3 \%$ & Brasil & $\mathbf{6 . 2 \%}$ \\
& República de Corea & $4.8 \%$ & México & $4.5 \%$ \\
\hline
\end{tabular}

Fuente: elaboración propia con base en los datos de Perfiles comerciales 2019, de la Organización Mundial del Comercio (omc).

Los datos anteriormente mencionados corroboran la hipótesis del poco dinamismo comercial entre ambas regiones sugerido a lo largo de este trabajo y reafirmado por el informe del BID. Es interesante mencionar que Chile es el miembro de la AP con mayor interacción comercial con el Mercosur. Ante esta situación, se entiende su papel al proponer de entrada la posible convergencia entre ambos bloques regionales.

A pesar de la ausencia de una alta interacción comercial, la convergencia entre ambos bloques resulta interesante ante la posible atracción de inversionistas regionales y extrarregionales, así como por la inserción en las cadenas globa- 
les de valor (CGV) ${ }^{55}$ Estos elementos son de interés para la AP, pues la colaboración con Brasil y Argentina, permitiría un acercamiento con China, socio estratégico de ambos países, para así proyectarse hacia la región del Asia-Pacifico. ${ }^{56}$

De esta manera, y a raíz del informe del diálogo de Alto Nivel entre Mercosur y la AP, en julio de 2018 los jefes de Estado de los países de ambos bloques concretaron el Plan de Acción de Puerto Vallarta donde, además de promover los elementos de la hoja de ruta, sumaron las intenciones de cooperación en materia de agenda digital, facilidades para invertir, comercio inclusivo y género, intercambio de experiencias en movilidad académica, turismo, cultura y movilidad de personas. ${ }^{57}$ Este pronunciamiento fue el último esquema referente a la convergencia de ambos bloques.

Tras la declaración del Plan de Puerto Vallarta, el 17 de julio de 2019, se lanzó el Comunicado conjunto de los Presidentes de los Estados Partes del Mercosur y los Estados Asociados, en el cual se destacó:

[L] os progresos alcanzados en el acercamiento entre el Mercosur y la Alianza del Pacifico, con base en el Plan de Acción de Puerto Vallarta. En este marco, elogiaron los esfuerzos tendientes a impulsar las sinergias, la articulación y la complementariedad de las agendas culturales del Mercosur y la Alianza del Pacífico. ${ }^{58}$

${ }^{55}$ Luz María de la Mora Sánchez, Hacia dónde se dirige el régimen de comercio internacional y sus implicaciones para América Latina, Cepal, 2018, p. 11, https://www.cepal.org/es/publicaciones/43621-donde-se-dirige-regimencomercio-internacional-sus-implicaciones-america-latina

${ }^{56}$ María M. Llairó, op. cit. p. 128.

${ }^{57}$ Plan de Puerto Vallarta, "Declaración presidencial de los países de la Alianza del Pacífico y del Mercosur", 2018, http:/ /www.cartillaciudadania.mercosur.int/oldAssets/uploads/Plan\%20de\%20Acción\%20-\% 20 Anexo\%20declaración\%20Puerto\%20Vallarta.pdf

${ }^{58}$ Ministerio de Relaciones Exteriores de Brasil, "Comunicado conjunto de los Presidentes de los Estados Partes del Mercosur y los Estados Asociados", Ministerio de Relaciones Exteriores de Brasil, 17 de julio de 2019, http://www.itamaraty.gov.br/es/notas-a-la-prensa/20641-comunicado- 
En suma, estos comunicados subrayan la intención de lograr la convergencia entre ambos bloques. No obstante, es necesario reconocer que los avances a los que hacen referencia se dirigen más hacia un ámbito político que a uno en materia económico-comercial, sobre todo por las escasas acciones encaminadas a incrementar el reducido intercambio comercial existente entre las partes.

\section{Adiós Macri. ¿EL Regreso Al DeSEntendimiento?}

A finales de 2019, Mauricio Macri es derrotado en las elecciones de Argentina ocasionando la salida de su gobierno de la Casa Rosada y el regreso de los peronistas. A primera vista, parecía que este nuevo cambio político sería un obstáculo a la postura aperturista que se había adoptado, en los últimos años, tanto en el gobierno argentino como en el Mercosur. Dicho estancamiento se materializó en las reuniones del Mercosur en abril a través de un anuncio donde se menciona que la República de Argentina dejaría de "participar en las negociaciones de los acuerdos comerciales en curso y de las futuras negociaciones del bloque, excluyendo de esta determinación a las ya concluidas con la Unión Europea y con la Asociación Europea de Libre Comercio". 59

No obstante, esta postura inicial del presidente argentino, Alberto Fernández, cambió drásticamente en la segunda mitad del 2020 cuando declaró que:

Guardo por todos los líderes el respeto que se merecen, aunque no pienso igual a muchos. Sé que mi paso por la historia argen-

conjunto-de-los-presidentes-de-los-estados-partes-del-mercosur-y-los-estados-asociados

${ }^{59}$ Ministerio de Relaciones Exteriores de Paraguay, "Mercosur: Argentina informó que dejará de participar de las negociaciones externas en curso", Ministerio de Relaciones Exteriores de Paraguay, 24 de abril de 2020, https: / www.mre.gov.py/index.php/noticias-de-embajadas-y-consulados/argentinadecide-retirarse-de-las-negociaciones-externas-que-emprende-el-mercosur 
tina es un paso y no tengo derecho a frustrar la aspiración continental que tenemos de unirnos y crecer juntos simplemente porque no pienso igual. Estoy aquí para que nos unamos. La historia nos ha dado la oportunidad de revisar lo que se ha hecho y hacerlo de otro modo. ${ }^{60}$

Esta declaración del presidente Fernández sugiere que sus ideales políticos no deben ser un impedimento al crecimiento y desarrollo en el continente. De esta manera, es posible establecer que continuará la visión de apertura del Mercosur, a pesar del cambio político que experimentó Argentina. Si bien pareciera que la aceptación de esta convergencia con la AP se moviliza por un factor económico ante los estragos que dejó la pandemia de Covid-19, es necesario considerar que esta situación extraordinaria modificó un comportamiento político que, de entrada, se mostraba dispuesto a cambiar las políticas implementadas por el gobierno anterior.

Finalmente, con respecto a la convergencia del Mercosur con la AP, en su última declaración conjunta, el 2 de julio de 2020, los presidentes expresaron "el interés en continuar trabajando entre el Mercosur y la Alianza del Pacifico, de acuerdo al Plan de Acción de Puerto Vallarta, con el fin de avanzar en los compromisos entre los dos bloques". ${ }^{61}$

\section{Consideraciones finales}

Se puede considerar que, a pesar de los comunicados oficiales que señalan los planes de acción en materia económica,

60 "Bipolaridad peronista en el Mercosur: Argentina cambia discurso una vez más", PanamPost, 10 de agosto de 2020, https://es.panampost. com/marcelo-duclos/2020/08/10/bipolaridad-peronista-mercosur-arg

${ }^{61}$ Ministerio de Relaciones Exteriores de Brasil, "Comunicado Conjunto de Presidentes de los Estados Partes del Mercosur y Estados Asociados-Asunción", Ministerio de Relaciones Exteriores de Brasil, 2 de julio de 2020, http://www.itamaraty.gov.br/es/notas-a-la-prensa/21600-comunicado-conjunto-de-presidente-dos-estados-partes-do-mercosul-e-estadosassociados-assuncao-2-de-julho-de-2021 
la convergencia entre el Mercosur y la AP se da principalmente en el ámbito político. Tal y como sugería la teoría, los mandatarios tienen gran capacidad de influencia en la aceptación o rechazo a los proyectos de integración, lo cual depende de la identificación de su política de gobierno. Para el caso examinado en esta investigación, el análisis de los gobiernos de Mauricio Macri y Jair Bolsonaro permitió entender cómo la influencia de estos dos gobiernos en el Mercosur dio paso a que este organismo fuera el instrumento clave para que se cumplieran las políticas de cada gobierno en pro de la liberalización comercial que se proponían.

La intención de convergencia entre ambos bloques fue posible por el cambio político que experimentaron Brasil y Argentina en sus gobiernos, pues el empate en sus ideas y la coordinación permitió que el Mercosur cambiará su postura hacia un organismo como la AP, pasando del rechazo en la época progresista a la aceptación y cooperación en la última década. Los procesos en torno a esta propuesta siguen desarrollándose, los avances en su ejecución no han logrado superar la fase discursiva que, hasta el momento, sólo ha permitido que los gobiernos de la región dieran a conocer los cambios en su política comercial con respecto al Mercosur por un lado y, por el otro, su aceptación a la apertura comercial de libre mercado.

Los cambios en los gobiernos de Argentina y Brasil ilustran el viraje que experimentó América Latina, sobre todo en una transición que apoya la liberalización económica por sobre un gobierno cerrado con crecimiento en el interior, para el caso argentino. Por su parte, el gobierno de Bolsonaro, pese a su postura antiglobalista, no muestra señales de poner freno a la apertura del Mercosur en un marco de libre comercio mientras ello le garantice el desarrollo que ha prometido a las bases que representa.

En lo que respecta al ámbito económico, el análisis sobre intercambio comercial permite sugerir que no hay elementos que marquen como prioritaria una alianza entre ambos bloques. No cabe duda que el mayor motor en el diálogo 
entre Mercosur y AP es el entendimiento político, todo lo cual se demostró con los gobiernos de los dos países más influyentes del Mercosur.

Los avances obtenidos hasta el momento han permitido que esta ambiciosa propuesta continúe siendo atractiva en la región. El progreso y la conclusión de este proceso estará determinado por el factor que cuente con mayor peso. Hasta el momento, la convergencia se ha basado en la búsqueda de progreso político, sin embargo, para un próximo análisis, cabe cuestionar si las situaciones extraordinarias, como la crisis del nuevo coronavirus (sARS-Cov-2) permitirá que el factor económico sea el nuevo determinante para reducir los estragos que ésta deje a su paso. Esperamos que el desarrollo de los hechos futuros permita dar respuesta a estos nuevos cuestionamientos.

\section{REFERENCIAS BIBLIOGRÁFICAS}

Acuerdo Marco de la Alianza del Pacífico, 17 de febrero de 2017, https://alianzapacifico.net/download/acuerdo-marco-de-laalianza-del-pacifico/

Alianza Pacífico, ¿Qué es la Alianza Pacífico?, https://alianzapacifico.net/que-es-la-alianza/

BACHELET, Michelle, "Discurso de S.E. La presidenta de la República, Michelle Bachelet, al inaugurar Seminario 'Diálogo sobre integración regional: Alianza del Pacífico y Mercosur'”, Dirección de prensa del Gobierno de Chile, 24 de noviembre de 2014, http:/ /archivospresidenciales.archivonacional.cl/uploads/r/ archivo-presidencia-de-la-acerepublica/6/6/b/66b5e5383881b 2187c106b989fa32d4feed0dac9e59689a27f7780d1201b4b07/_ home_aristoteles_documentos_DIS_0350.pdf

"Bipolaridad peronista en el Mercosur: Argentina cambia discurso una vez más", PanamPost, 10 de agosto de 2020, https://es.pa nampost.com/marcelo-duclos/2020/08/10/bipolaridad-pe ronista-mercosur-arg 
Bоввіо, Norberto, Derecha e izquierda: razones y significados de una distinción política, Madrid, Taurus, 1996.

Briceño Ruiz José, "Estado, mercado y el modelo económico de la integración regional en América Latina, los casos de Mercosur y Alianza del Pacífico”, en José Briceño Ruiz y Edgar Viera Posada (eds.), Repensar la integración en América Latina, Colombia, Universidad Cooperativa de Colombia, 2019, pp. 21-68.

Busso Anabella y Julieta ZeLicovich, "El gobierno de Mauricio Macri y la integración regional: ¿desde el Mercosur a la Alianza del Pacífico?”, Conjuntura Austral, Vol. 7, núm. 37, 2016, pp. 17-24, https://doi.org/10.22456/2178-8839.66725

Castaño, Francisco, "La inserción internacional argentina durante el gobierno de Macri”, ponencia presentada en el VIII Encuentro del CERPI y la VI Jornada del Censud, unLP, Argentina, 2017, 25 pp., http://sedici.unlp.edu.ar/handle/10915/64890

Castro Madureira Vinícius de Carvalho y Carolina P. de Souza, "Brasil y la integración regional", Estado E゚ Comunes, Revista de políticas y problemas públicos, vol. 2, núm. 9, 2019, pp. 199-210, https://doi.org/10.37228/estado_comunes.v2.n9.2019.125

Chabay, Ezequiel, "Guedes, en exclusiva: qué pasa con el "peso real', por qué votaría a Macri y qué pasará si vuelve CFK”, El Cronista, 16 de julio de 2019, https://www.cronista.com/economiapolitica/Guedes-en-exclusiva-que-pasa-con-el-peso-real-por-que-vo taria-a-Macri-y-que-pasara-si-vuelve-CFK-20190716-0084.html

Chuburu, Daniel, "La convergencia Mercosur, Alianza del Pacífico desde la perspectiva argentina: los desafíos de profundizar la integración regional en la actualidad", en Mariana Aparicio Ramírez y Tomás Milton Muñoz Bravo, Retos y oportunidades de la Alianza del Pacífico en el actual contexto internacional convulso, México, unam, 2019, pp. 41-48.

Clemente Batalla, Isabel, "Comercio y protección externa de Uruguay en la Cuenca del Pacífico", en Mariana Aparicio Ramírez y Tomás Milton Muñoz Bravo, Retos y oportunidades de la Alianza del Pacifico en el actual contexto internacional convulso, México, UnAm, 2019, pp. 155-171.

"Chile y Paraguay impulsan la relación Mercosur/Alianza del Pacífico”, MercoPress, Montevideo, abril de 2018, https://es.mer 
copress.com/2018/04/21/chile-y-paraguay-impulsan-la-rela cion-mercosur-alianza-del-pacifico

Clemente Batalla Isabel, Camilo López Burian y Diego Telias, "Uruguay y la Alianza del Pacífico: ¿repensando el modelo de inserción internacional?”, Cuadernos sobre Relaciones Internacionales, Regionalismo y Desarrollo, Venezuela, vol. 10, núm. 19, 2015, pp. 23-46, http://www.saber.ula.ve/bitstream/handle/1234567 89/42573/articulo2.pdf;jsessionid=681C8959E5C3DE4BB816 C0C1D74D118B? sequence $=1$

D’Alesio, Laura María, "La 'nueva derecha' y su política exterior. Los primeros tres años de gestión del gobierno de Mauricio Macri”, Perspectivas Revista de Ciencias Sociales, año 4, núm. 8, 2019, pp. 194-223, https://doi.org/10.35305/prcs.v0i8.58

Delegation of the European Union to Chile, "La UE y el Mercosur llegan a un acuerdo en materia de comercio", Bruselas, Comisión Europea, 28 de junio de 2019, https://eeas.europa.eu/ delegations/chile/64865/la-ue-y-el-mercosur-llegan-un-acuer do-en-materia-de-comercio_uz

Frenkel, Alejandro y Diego Azzi, "Cambio y ajuste: la política exterior de Argentina y Brasil en un mundo en transición (20152017)", Colombia Internacional, núm. 96, 2018, pp. 177-207, https://revistas.uniandes.edu.co/doi/pdf/10.7440/colom biaint 96.2018 .07

Gobierno de Argentina, "Macri, sobre el acuerdo Mercosur-uE: 'Es fruto de ese trabajo colectivo'”, Argentina, Casa Rosada Presidencia, 2019, https://www.casarosada.gob.ar/slider-principal/ 45831-macri-sobre-el-acuerdo-mercosur-ue-es-fruto-de-ese-tra bajo-colectivo

Hernández, René, "La encrucijada de la integración regional y el nuevo regionalismo", Revista Estudios de Políticas Públicas, vol. 7, 2018, pp. 149-159, https://doi.org/10.5354/0719-6296.2018.5 0427

InOTAI Andras, "Regional integration among developing countries, revisited”, Policy Research Working Paper Series, núm. 643, The World Bank, 1991, 56 pp., http://documents1.world bank.org/curated/en/647131468739252693/pdf/multi0pa ge.pdf 
Instituto para la Integración de América Latina y el Caribe, Mercosur-Alianza del Pacífico. Informe del Diálogo de Alto Nivel: una agenda positiva para la integración, BID, marzo de 2019, https:// publications.iadb.org/es/publicacion/14106/mercosur-alian za-del-pacifico-informe-del-dialogo-de-alto-nivel-una-agenda

KLAVEREN, Alberto van, "El eterno retorno del regionalismo latinoamericano", Revista Nueva Sociedad, núm. 275, 2018, pp. 62-72, https://nuso.org/media/articles/downloads/3.TC_van_Kla veren_275.pdf

"La Unión Europea y el Mercosur logran un acuerdo comercial tras veinte años de negociación", Agencia EFE, 28 de junio de 2019, https://www.efe.com/efe/america/portada/la-unioneuropea-y-el-mercosur-logran-un-acuerdo-comercial-tras-veinte-anos-de-negociacion/20000064-4011791

Langenhove, Luk, Building Regions: The Regionalization of the World Order, Farnham, Ashgate, 2011.

Langhammer Rolf J. y Ulrich Hiemenz, "Regional integration among developing countries: opportunities, obstacles and options”, Kieler Studien, núm. 232, 1990, 103 pp., https:/ /www.eco nstor.eu/handle/10419/416

Llairó, María de Monserrat, "Los nuevos desafíos y ejes de poder de la integración latinoamericana: la dualidad Mercosur-Alianza del Pacífico (2010-2017)", Anuario Latinoamericano-Ciencias Políticas y Relaciones Internacionales, vol. 7, 2019, pp. 111-129, https://doi.org/10.17951/al.2019.7.111-129

Martínez-Castillo, Alberto, "The Pacific Alliance and Mercosur: Two Approaches to Economic Regional Integration", Papel Politico,vol.21, núm. 2, 2016, pp.469-494, https://doi.org/10.11144/ Javeriana.papo21-2.apmc

Mercosur, ¿Qué es el Mercosur?, https:/ / www.mercosur.int/quienessomos/en-pocas-palabras/

Mercosur, Protocolo de Ushuaia sobre Compromiso Democrático en el Mercosur, 24 de julio de 1998, https://www.mercosur. int/documento/protocolo-ushuaia-compromiso-democrati co-mercosur-bolivia-chile/

Ministerio de Relaciones Exteriores de Brasil, "Comunicado conjunto de los Presidentes de los Estados Partes del Mercosur y 
los Estados Asociados", Gobierno de Brasil, 17 de julio de 2019. http:/ / www.itamaraty.gov.br/es/notas-a-la-prensa/20641-co municado-conjunto-de-los-presidentes-de-los-estados-partesdel-mercosur-y-los-estados-asociados

Ministerio de Relaciones Exteriores de Brasil, "Declaración conjunta emitida en el marco de la visita de trabajo a Brasil del Presidente de la Nación Argentina, Mauricio Macri-Brasilia”, 16 de enero de 2019, Gobierno de Brasil, 16 de enero de 2019, http:/ / www.itamaraty.gov.br/es/notas-a-la-prensa/19964-declaraci on-conjunta-emitida-en-el-marco-de-la-visita-de-trabajo-a-brasildel-presidente-de-la-nacion-argentina-mauricio-macri-brasilia16-de-enero-de-2019

Ministerio de Relaciones Exteriores de Brasil, "Declaración Conjunta Presidencial en ocasión de la visita de Estado del Presidente Jair Bolsonaro a Buenos Aires", Gobierno de Brasil, 6 de junio de 2019, http:/ / www.itamaraty.gov.br/es/notas-a-la-prensa/204 83-declaracion-conjunta-presidencial-en-ocasion-de-la-visita-deestado-del-presidente-jair-bolsonaro-a-buenos-aires

Ministerio de Relaciones Exteriores de Brasil, "Comunicado Conjunto de Presidentes de los Estados Partes del Mercosur y Estados Asociados - Asunción”, Gobierno de Brasil, 2 de julio de 2020, http:/ / www.itamaraty.gov.br/es/notas-a-la-prensa/21600comunicado-conjunto-de-presidente-dos-estados-partes-domercosul-e-estados-associados-assuncao-2-de-julho-de-2021

Ministerio de Relaciones Exteriores de Paraguay, Mercosur: Argentina informó que dejará de participar de las negociaciones externas en curso, Gobierno de Paraguay, 24 de abril de 2020, https:/ / www. mre.gov.py/index.php/noticias-de-embajadas-y-consulados/ argentina-decide-retirarse-de-las-negociaciones-externas-queemprende-el-mercosur

Mora SÁnchez, Luz María de la, Hacia dónde se dirige el régimen de comercio internacional y sus implicaciones para América Latina, Cepal, 2018, 130 pp., https://www.cepal.org/es/publicaciones /43621-donde-se-dirige-regimen-comercio-internacional-susimplicaciones-america-latina

Moreira, Constanza, "El largo ciclo del progresismo latinoamericano y su freno: los cambios políticos en América Latina de la 
última década (2003-2015)", Revista Brasileira de Ciências Sociais, Brasil, vol. 32, núm. 93, 2019, pp. 1-28, http://www.scielo.br/ pdf/rbcsoc/v32n93/0102-6909-rbcsoc-3293112017.pdf

Organización Mundial del Comercio, omc, Perfiles comerciales 2019 , OMC, 2019, https://www.wto.org/spanish/res_s/booksp_s/tra de_profiles19_s.pdf

Pastrana, Eduardo y Rafael Castro, "La Alianza del Pacífico, un eje articulador del interregionalismo pos-TPP", Anuario de la integración regional de América Latina y el Caribe, núm. 14, 2017, pp.71-95,http://www.cries.org/wp-content/uploads/2018/04/ Anuario-2017-final.pdf

Plan de Puerto Vallarta, Declaración presidencial de los países de la Alianza del Pacífico y del Mercosur, 2018, http://www.carti llaciudadania.mercosur.int/oldAssets/uploads/Plan $\% 20$ de\%20Acción\%20-\%20Anexo\%20declaración\%20Puerto\% 20Vallarta.pdf

Rodrigues, Gilberto, “¿El Trump del trópico? Política exterior de ultraderecha en Brasil”, Análisis Carolina, núm. 6, 2019, pp. 1-11, https://doi.org/10.33960/AC_06pt.2019

Rodríguez Minor, Rebeca, "Alianza del Pacífico vs. Mercosur. ¿Integración o exclusión regional?”, Revista de Relaciones Internacionales, núm. 125, 2016, pp. 119-144, http://www.revistas. unam.mx/index.php/rri/article/view/58600

SanahuJa, José Antonio, "El ascenso global de la ultraderecha y el nacionalismo: Crisis de la globalización, el regionalismo y el orden liberal", en Josette Altmann Borbón (ed.), América Latina frente a la reconfiguración global, Costa Rica, Facultad Latinoamericana de Ciencias Sociales, 2019, pp. 31-64.

Schiff, Maurice y Alan L. Winters, Regional integration and development, Washington, D.C., World Bank Group, 2003, 321 pp., http:/ / documents.worldbank.org/curated/en/26368146878 0873762/Regional-integration-and-development

Schneider, Christina J., "The Political Economy of Regional Integration”, Annual Review of Political Science, vol. 20, 2017, pp. 229-248, https://www.annualreviews.org/doi/full/10.1146/annurev-po lisci-051215-023006 
VAdell Javier A. y Clarisa Giaccaglia, "El rol de Brasil en el regionalismo latinoamericano: la apuesta por una inserción internacional solitaria y unilateral", Foro Internacional, vol. 60, núm. 3, 2020, pp. 1041-1080, https://doi.org/10.24201/fi.v60i3.2770 
